\title{
Nefrectomía laparoscópica mano-asistida
}

\author{
Gutiérrez Sanz-Gadea C, Mus Malleu A, Briones Mardones G, Hidalgo Pardo F, Rebassa Llull M, \\ Conde Santos G.
}

Servicio de Urología. Hospital Son Llàtzer. Palma de Mallorca

Actas Urol Esp. 2006;30(7):698-706

\begin{abstract}
RESUMEN
NEFRECTOMÍA LAPAROSCÓPICA MANO-ASISTIDA

Introducción y Objetivos: Presentamos nuestra casuística de nefrectomías laparoscópicas mano-asistidas. Se valoran nuestros resultados y las dificultades del inicio de un programa progresivo de laparoscopia.

Material: Desde noviembre 2003 hasta noviembre 2005 se han realizado 35 nefrectomías laparoscópicas Mano-asistidas, de un total de 45 nefrectomías laparoscópicas. 22 nefrectomías radicales, 3 nefrectomías simples y 10 nefroureterectomías. Media de edad 66 años (47-89). Tenian una media de sobrepeso (IMC 28,3); 38\% eran obesos. ASA 2,3 (1-4). Los tumores tenian $4,8 \mathrm{~cm}$ de tamaño medio; $80 \%$ eran T1. El 28,6\% habían sido operados previamente de cirugía abdominal.

Resultados: Se realizaron 1 en 2003, 17 en 2004, y 17 en 2005. El tiempo quirúrgico ha sido 140 minutos, 130 minutos (80-210) en nefrectomías radicales, 135 minutos (120-150) en nefrectomías simples y 163 minutos (80-240) en nefroureterectomías. Los pacientes iniciaron dieta oral en 1,7 días y se levantaron en 1,7 días. Los obesos iniciaron la dieta ( 2,3 días) y se levantaron (2,4 días) más tarde que los no obesos (1,5 y 1,4 días respectivamente). La estancia media ha sido 5,8 días (3-15); en los no complicados 4,2 días y en los que han tenido complicaciones 9,7 días. En ningún caso se trasfundió. Hemos tenido 11,4\% de complicaciones mayores con EM de 11,7 días. Un 5,7\% de reconversiones y 5,7\% de reoperaciones. Y 17,1\% de complicaciones menores, con EM de 8,8 días.

Conclusiones: La nefrectomía mano-asistida es una técnica que por sus ventajas permite un inicio en la laparoscopia, con menor curva de aprendizaje, y resultados satisfactorios, permitiendo incorporar la cirugía laparoscópica a hospitales con un menor volumen anual.
\end{abstract}

Palabras clave: Nefrectomía. Laparoscopia. Cáncer renal. Nefrectomía mano-asistida.

\section{ABSTRACT}

HAND-ASSISTED LAPAROSCOPIC NEPHRECTOMY

Introduction and objectives: To present the number of cases of hand-assisted laparoscopic nephrectomy. This study evaluates our results and difficulties of starting a progresive programme of laparoscopy.

Material and Method: Between november 2003 and november 2005, 35 hand-assisted laparoscopic nephrectomies were carried out, of a total of 45 laparoscopic nephrectomies. 22 radical nephrectomies, 3 simple nephrectomies and 10 nephroureterectomies. The average age of patients was 66 years (47-89). On average, they were overweight (IMC 28,3), with approximately $38 \%$ being obese. ASA 2.3 (1-4). The tumors measured $4.8 \mathrm{~cm}$ on average, and $80 \%$ of these were T1. In $28.6 \%$ of the cases, they had previously undergone abdominal surgery.

Results: One was undertaken in 2003, 17 in 2004, and 17 in 2005. The surgery time was 140 minutes, 130 minutes (80-210) in radical nephrectomies, 135 minutes (120-150) in simple nephrectomies and 163 minutes (80-240) in the nephroureterectomy. Patients began an oral diet 1.7 days later, and were able to get up 1.7 days later. In the case of obese patients, they began an oral diet 2.3 days later and were able to get up (2.4 days) later than those non obese cases. (1.5 and 1.4 days respectively). The average stay has been 5.8 days (3-15). The average stay of the cases without complications was 4.2 days and those that encountered complications 9.7 days. In no cases was there a need for a blood transfusion. In $11.4 \%$ we had major complications with an average stay of 11.7 days. In $5.7 \%$ of cases there were reconversions, and $5.7 \%$ of cases were reoperated. $17.1 \%$ had minor complications, with an average stay of 8.8 days

Conclusions: The advantage of hand-assisted nephrectomy is that it allows one to begin a laparoscopy, with a reduced learning time, and with satisfactory results, allowing the incorporation of laparoscopy surgery in hospitals with a reduced annual volume.

Keywords: Nephrectomy. Laparoscopy. renal cancer. Hand-assisted nephrectomy. 
L a utilización de la laparoscopia para realizar una nefrectomía fue publicada por primera vez por Clayman en 1991 en un oncocitoma'; desde entonces se abrió un nuevo abordaje terapéutico, que ha llevado a considerar la nefrectomía laparoscópica como el "gold standard" en la extirpación renal. Inicialmente se realizaban nefrectomías simples; luego se han realizado todo tipo de nefrectomías ${ }^{2}$.

Con la laparoscopia se ha conseguido reducir el dolor postoperatorio, la estancia hospitalaria, una mayor recuperación del paciente, con incorporación más rápida a su vida habitual, y evidente mejoría cosmética. Y todo ello, sin menoscabar el resultado del tratamiento, tanto de las nefrectomias por enfermedades renales benignas, como los resultados oncológicos en el carcinoma renal y en los tumores uroteliales. La indicación de la técnica laparoscópica está influenciada por la experiencia del cirujano, y su confianza en la técnica. Nosotros decidimos incorporar la nefrectomía laparoscópica Mano-asistida por creer que nos permitiría acceder a mayores indicaciones de laparoscopia, con menor riesgo, menor curva de aprendizaje, pudiendo por ello ser utilizada por un mayor número de miembros del servicio, dado el número limitado de pacientes que se pueden realizar cada año. Actualmente en caso de riñones pequeños realizamos la nefrectomía laparoscópica convencional.

La laparoscopia Mano-asistida es una técnica mínimamente invasiva combinando la laparoscopia con la introducción de la mano no dominante del cirujano para facilitar la operación, añadiendo muchas posibilidades. También puede ser el ayudante el que introduzca la mano y el cirujano emplee ambas con los instrumentos laparoscópicos. Para ello se han diseñado varios dispositivos desde 1997 en que se introdujo el Pneumo Sleeve: Inicialmente los de primera generación, el Pneumo Sleeve, el Hand Port, el Intromit y posteriormente los de segunda generación: LapDisc, Omniport y Gelport ${ }^{3,4}$.

En 1993, Boland et al., publicaron su experiencia ayudándose de un guante en el abdomen para facilitar la laparoscopia ${ }^{5}$. En 1996 se publicó la primera nefrectomía laparoscópica manoasistida en modelo porcino ${ }^{6}$. La primera nefrectomía laparoscópica Mano-asistida en humanos con éxito la publicaron en 1997 Nakada et al. ${ }^{7}$, y ese mismo año se comunicó una nefroureterectomía Mano-asistida ${ }^{8}$. Desde entonces la laparoscopia mano-asistida se ha utilizado en nefrectomía simple y nefrectomía radical $^{9}$, en nefroureterectomía ${ }^{8,10,11}$, nefrectomía del donante vivo $^{12}$, nefrectomía parcial ${ }^{13-15}$, cistectomía radical $^{16}$, etc...

En 1998 Wolf et al. mostraron la similitud de resultados entre nefrectomía laparoscópica Mano-asistida y nefrectomía laparoscópica ya llamada entonces "standard"17; y Nakada en 1999 describió la técnica quirúrgica ${ }^{18,19}$.

Analizando varias publicaciones sobre ventajas de la manoasistida ${ }^{17,18,20-22}$, se pueden citar:

- Técnica más rápida y más sencilla: permite iniciar la laparoscopia en hospitales con menor casuística.

- Acceso más controlado y seguro de los trócares en casos complejos, multioperados.

- Menor curva de aprendizaje.

- Menor estrés para el equipo quirúrgico.

- Convierte la mano en otro instrumento permitiendo el tacto, y la disección roma digital, así como la tracción de los tejidos y la separación con la mano o los dedos.

- Se pueden aumentar las indicaciones de laparoscopia en casos más difíciles, por tener tejidos inflamatorios, multioperados, muy adheridos, o en tumores grandes que de otra forma se realizaría un abordaje abierto.

- Permite la introducción de otros instrumentos gruesos a su través.

- Facilita el control de complicaciones, al ayudarse de la mano para su resolución.

- Utiliza al final el puerto Mano-asistido para extraer el órgano, por ello en las piezas grandes no altera la estética de la laparoscopia convencional. Hay que tener en cuenta que para extraer la pieza de nefrectomía en un riñón no atrófico, y más si está agrandado por ser tumoral se precisa una incisión de al menos 5-6 cm, y con el puerto Mano-asistido se precisan 6-8 cm según el tamaño de la mano del cirujano ${ }^{23}$.

- Incluso puede evitar reconvertir una laparoscopia inicial con complicaciones intraoperatorias, colocando un puerto Mano-asistido para resolverlas ${ }^{17}$. 
También tiene inconvenientes ${ }^{18,24}$ (algunos han mejorado con los nuevos equipos):

- Es un dispositivo caro.

- Exige menos habilidades técnicas, por ello el cirujano se puede acomodar y no progresar en la técnica laparoscópica tan necesaria en otras intervenciones laparoscópicas.

- En algunos accesos se llega mal con la mano.

- Hay más fugas de gas y por ello más consumo.

- Posición menos ergonómica para el cirujano y por ello mayor cansancio quirúrgico (compensado por la mayor rapidez).

\section{RESULTADOS}

Del total de 45 nefrectomías laparoscópicas realizadas en nuestro servicio desde marzo 2003 a noviembre 2005, se presentan 35 (el 77,8\%) que se han realizado Mano-asistidas; en 9 se ha realizado nefrectomía laparoscópica convencional y en 1 nefrectomía parcial laparoscópica. Se han realizado 22 nefrectomias radicales por carcinoma renal, 10 nefroureterectomías y 3 nefrectomías por patologías benignas.

La vía de acceso del puerto de Mano-asistida se muestra en las Figuras 1 y 2 en el lado derecho una incisión de McBurney y en el lado izquierdo una incisión media supraumbilical o pararrectal. Después de introducir la mano se colocan los puertos bajo control de un dedo; a veces hemos introducido un trocar a través del Gelport para iniciar el neumoperitoneo, o la cámara para ver la cavidad antes de introducir los trócares.

Los pacientes tenían una media de edad de 66 años (47-89); eran 25 varones y 10 mujeres. Inicialmente, empezamos usando el OmniPort (20 casos), pero desde agosto 2004 se ha utilizado principalmente el GelPort (15 casos) (Fig. 3).

Estas operaciones han sido realizadas por 3 urólogos, inicialmente empezamos 2 y luego se incorporó un tercero, participando otros 3 urólogos como ayudantes.
Se hicieron 1 en 2003, 17 en 2004 (el 57\% de las nefrectomías), y 17 en 2005 (el 83\% del total) (Fig. 4).

Se ha determinado el ASA (American Society of Anesthesiologists score) de los pacientes, siendo una media de 2,3 (1-4); y el IMC (Índice de Masa Corporal), con una media de 28,3 (21,9-40) considerada como sobrepeso, lo que corresponde a un peso medio de $81 \mathrm{~kg}$ (50-124), con 38\% de Obesidades (IMC de 30 o superior).

\section{Cirugía abdominal previa}

El $28,6 \%$ habían sido operados de cirugía abdominal $(11,4 \%$ apendicectomía, 11,4\% colecistectomía abierta, 5,7\% herniorrafia y 11,4\% otras técnicas).

\section{Enfermedades concomitantes}

El 34,3\% de los pacientes recibían antiagregación plaquetaria o anticoagulación por vasculopatías o cardiopatías, 14,3\% tenían cardiopatía (5,7\% IAM), 14,3\% HTA, 11,4\% hernia hiato,

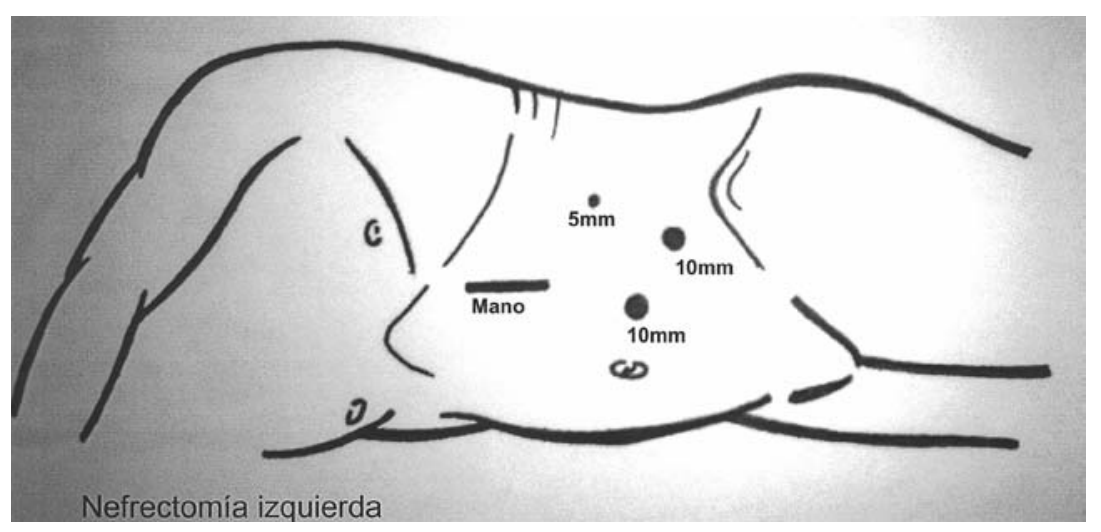

FIGURA 1

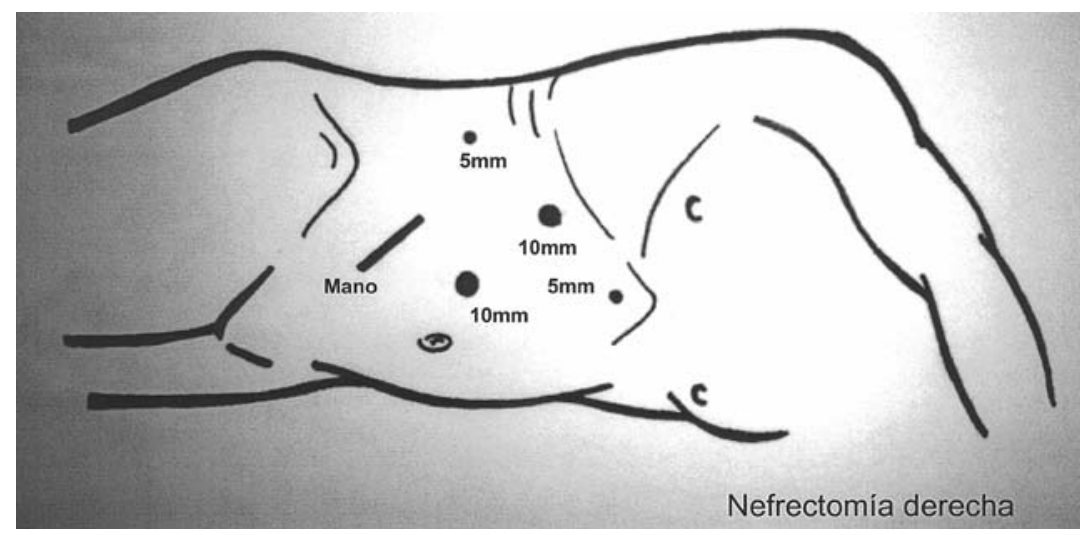

FIGURA 2 


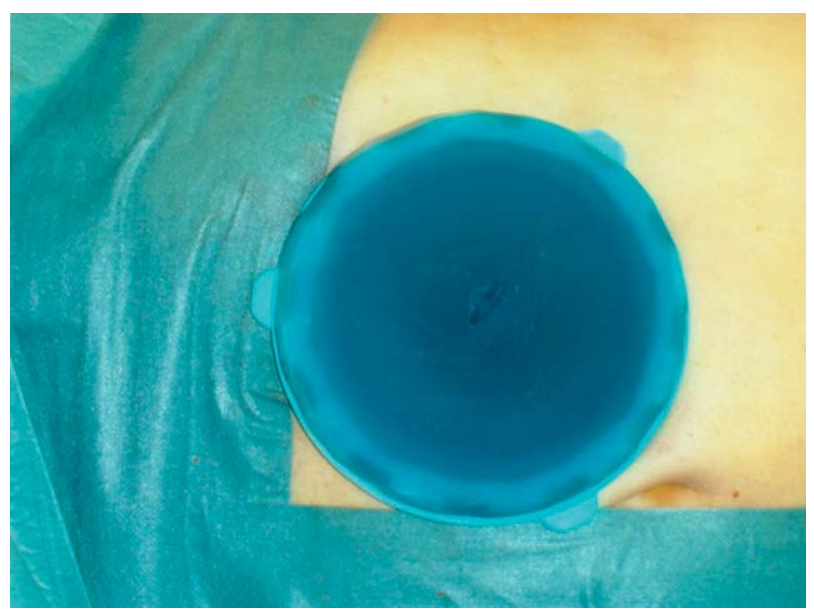

FIGURA 3

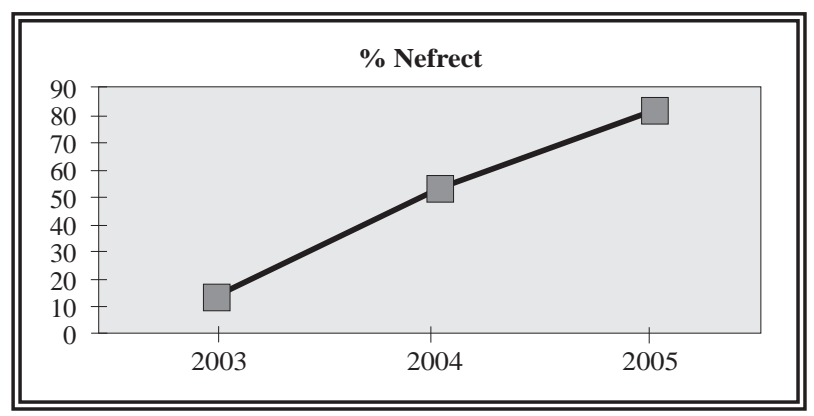

FIGURA 4

$8,6 \%$ asma/EPOC, 5,7\% pancreatitis aguda, 2,9\% ACVA, 2,9\% $\mathrm{HIV}+, 2,9 \%$ habian recibido radioterapia pelviana por carcinoma vesical, 2,9\% aneurisma de aorta, 2,9\% tenían marcapasos cardíaco, y 2,9\% insuficiencia renal crónica.

\section{Datos histopatológicos}

Se extrajo una pieza quirúrgica de 490 g (195896) en las nefrectomías radicales, 460 g (147853) en las nefroureterectomías y 312 g (50-626) en las nefrectomías simples.

De los carcinomas renales se obtuvieron tumores de tamaño histopatológico de $4,8 \mathrm{~cm}$ de media $(2,3-8,5)$. El estadiaje histopatológico de los carcinomas de células claras ha sido: $16 \mathrm{~T} 1$ (el 80\%), 2 T2, 2 T3b, y hemos tenido 1 quiste calcificado y un nefroma quístico.

\section{Datos quirúrgicos}

El tiempo quirúrgico ha sido 140 minutos: 130 (80-210) en las nefrectomías radicales, 135 (120150) en las nefrectomías simples y 163 (80-240) en las nefroureterectomías (tiempo laparoscópico, habitualmente se hace desinserción ureteral endoscópica, excepto 1 caso en que se hizo abierta al final, por tener tumor en el orificio ureteral, y otro que se dejó el uréter intravesical por tener 80 años y tejido pelviano radiado).

\section{Datos postoperatorios}

No se ha valorado el dolor, pues todos los pacientes, a criterio del servicio de anestesia, han llevado bomba de analgesia peridural desde la operación, durante 1-2 días.

Los pacientes han iniciado dieta oral al día siguiente en el 49\% y se levantaron al día siguiente en el 40\%. Iniciaron dieta oral en 1,7 dias y deambularon en 1,7 días. Los obesos iniciaron la dieta oral (2,3 dias) y se levantaron (2,4 dias) más tarde que los no obesos $(1,5$ y 1,4 días respectivamente), sin guardar relación con la edad ni con el ASA.

La estancia media (EM) ha sido 5,8 días (315), estando el 40\% 3-4 días. Los motivos de las estancias más prolongadas han sido: Una lesión arterial mayor con reimplante, íleo paralítico con drenaje linfático prolongado, $y$ fallo en la EndoGIA con reconversión. Tenemos EM mayor en las nefroureterectomías. El inicio de ingesta oral postoperatoria fue de 1 día en las nefrectomías simples, 1,7 en las nefrectomias radicales y 2 en las nefroureterectomías.

\section{Complicaciones}

La EM en los no complicados ha sido 4,2 días y en los que han tenido complicaciones 9,7. En ningún caso se trasfundió, aunque hubo descensos de la hemoglobina de $2,8 \mathrm{~g} / 1$ de media (de $13,9$ a 11,1$)$.

\section{Complicaciones mayores}

Hemos tenido un $11,4 \%$. 5,7\% (2) reconversiones, una por fallo en la EndoGIA estando cerrada sobre la vena renal derecha, y en otro paciente con tumor en cara medial renal izquierda, sobre la aorta, una ligadura de la arteria mesentérica superior. Y 5,7\%(2) reoperaciones: un sangrado venoso de la pared lumbar en un caso muy adherido, inflamatorio, con piohidronefrosis, que pasó desapercibida durante la intervención, y un drenaje de hematoma al $4^{\circ}$ día postoperatorio, estando ya en $\mathrm{su}$ domicilio. En estos casos la EM ha sido 11,7 días. 
Complicaciones menores: En un 17,1\% (6 pacientes), con EM de 8,8 días:

2 Drenajes prolongados, uno con íleo paralítico secundario.

2 Íleos paralíticos (uno con obesidad mórbida, IMC 40).

2 Dolores, uno abdominal y otro frénico (ambos muy obesos, IMC superior a 30)

1 Descompensación con insuficiencia cardíaca (ASA 3 con muchas patologías asociadas).

\section{DISCUSIÓN}

En el planteamiento de la incorporación de la laparoscopia en nuestro servicio se ha intentado tener un proyecto de participación de los miembros del servicio desde el inicio, por ello las nefrectomías laparoscópicas han sido realizadas por 3 urólogos, inicialmente 2 y luego se ha incorporado un tercero. Así el 37,5\% (30-43\% según épocas) de los urólogos han participado como cirujanos en las nefrectomías laparoscópicas. Además para la difusión de la técnica por todo el servicio, han participado 3 miembros más como ayudantes en un tercio de los casos. La presencia de 3 urólogos como cirujanos es dificil de valorar, pues se mantiene una permanente curva de aprendizaje, cada vez menos importante, que seguramente influirá en los resultados. En la literatura no siempre se refiere, pero oscila entre 1-5 cirujanos ${ }^{25}$.

El aprendizaje de la laparoscopia mano-asistida es rápido, y más fácil que en la laparoscopia convencional, incluso aunque en inicio es más dificil que la nefrectomía abierta, a los pocos casos se considera una intervención de menor dificultad $^{22}$. La curva de aprendizaje va reduciéndose a medida de la incorporación de nuevos miembros en el equipo, como subjetivamente nos ha parecido en nuestra experiencia.
En la nefrectomía laparoscópica Mano-asistida retroperitoneal son necesarias sólo 5-10 intervenciones para adquirir experiencia, recomendando no pasar a la laparoscopia convencional hasta haber realizado 10 Mano-asistidas ${ }^{26}$. En un análisis de formación en laparoscopia durante la residencia en urología se ha evidenciado una menor curva de aprendizaje con la Mano-asisti$\mathrm{da}^{22}$, y se puede realizar con poca experiencia en laparoscopia ayudado por un tutor ${ }^{27}$.

Gracias a la utilización de la Mano-asistida en nuestro servicio se han reducido mucho las indicaciones de nefrectomía abierta convencional (Fig. 4): en 2003 hicimos el 17\% de las nefrectomías por laparoscopia, en el año 2004 el 57\% y en 2005 el $83 \%$, (Fig. 4) por lo que consideramos la nefrectomía laparoscópica el procedimiento "standard" en nuestro servicio desde 2004. En los casos de nefrectomía por causa inflamatoria, al ser más complejos es más útil la Mano-asistida ${ }^{28}$.

Las Tablas 1 y 2 muestran los resultados comparativos de las nefrectomías Mano-asistidas.

No hemos establecido una edad límite para su indicación. La edad media de nuestros pacientes es 66 años (47-89), similar a la de la literatura, teniendo $11,4 \%$ pacientes mayores de 80 años $(34,3 \%$ de 75 o más). Y ante la baja morbilidad y precoz recuperación nos ha animado a indicar la cirugia en pacientes mayores, pues quizá la técnica abierta hubiera sido para ellos muy compleja. Cobb et al. revisan sus nefrectomías laparoscópicas en mayores y menores de 65 años, sin diferencias en tiempo quirúrgico, sangrado, ni en complicaciones, sólo encontrando una leve mayor $\mathrm{EM}^{29}$. Ellos tienen un $35 \%$ de mayores de 65 años ${ }^{29}$; en nuestra serie es del $51 \%$.

Tabla 1

Tipo de pacientes y resultados postoperatorios de nefrectomías Mano-asistidas

\begin{tabular}{|c|c|c|c|c|c|c|c|c|c|c|}
\hline & Tipo de Op & No & Edad & $\begin{array}{l}\text { Tiempo } \\
\text { Op(min) }\end{array}$ & IMC & ASA & $\begin{array}{c}\text { Dieta } \\
\text { Oral(dias) }\end{array}$ & $\begin{array}{l}\text { Anda } \\
\text { (dias) }\end{array}$ & EM & $\begin{array}{c}\text { Tamaño } \\
\text { (cm) }\end{array}$ \\
\hline Nosotros & & 35 & 66 & 140 & 28,3 & 2,3 & 1,7 & 1,7 & 5,8 & 4,8 \\
\hline Patel y Leveille ${ }^{36}$ & Nef Radical (T1) & 50 & 62 & 190 & & 1,9 & & & 3,3 & 4,7 \\
\hline Nelson y Wolf 30 & Nef Radical & 22 & 57 & 205 & 29,1 & 2,2 & 0,5 & 1,3 & 2,7 & 6,3 \\
\hline Landman et al. ${ }^{37}$ & Nefroureterectomía & 16 & 66 & 294 & & 2,7 & 0,5 & & 2,5 & \\
\hline López et al. ${ }^{35}$ & Nef Radical & 11 & 61 & 156 & 28,5 & 2,1 & & & 3,1 & 5,5 \\
\hline Kawauchi et al. ${ }^{11}$ & Nefroureterectomía & 34 & 67 & 233 & 21,6 & & 1,8 & 1,5 & 13 & \\
\hline Pietrow et al. ${ }^{38}$ & & 36 & 55 & 175 & 28,6 & 2,3 & 1 & & 4,3 & 4,3 \\
\hline Harano et al. ${ }^{34}$ & Nef Radical & 96 & 61 & 246 & & & 1,7 & 1,7 & 11,4 & 4,3 \\
\hline
\end{tabular}


Hemos utilizado tanto el Omniport como el Gelport, no encontrando diferencias especiales, aunque preferimos en la actualidad el Gelport, al considerar que tiene ventajas, como la posibilidad de salir y entrar sin perder el neumoperitoneo, permite intercambiarse los cirujanos, cambiar de posición, participar más urólogos, lo que mejora la curva de aprendizaje, poder introducir instrumentos a su través como otro puerto laparoscópico, algunos incluso gruesos, se puede convertir en un puerto para el paso de pinzas. Tiene una mayor comodidad para el cirujano, pues comprime menos la mano. En el estudio comparativo de Patel y Stifelman acerca de los dispositivos para Mano-asistida, refieren que el Gelport es el más satisfactorio entre todos los cirujanos que lo utilizaron ${ }^{4}$.

Hay series de laparoscopia en que el $21 \%^{11}$, la $\operatorname{mitad}^{25}$, o el $63,6 \%{ }^{30}$ tenían cirugías abdominales previas. El 28,6\% de nuestros pacientes habian sido operados previamente de cirugía abdominal no ocasionándonos ningún problema, comprobando que la cirugía previa no es una contraindicación. Sólo en un caso se desestimó la laparoscopia en un paciente muy complejo, portador de colostomía por diverticulitis con peritonitis, reoperaciones múltiples por abscesos abdominales y eventraciones, con múltiples mallas y persistencia de la eventración, por lo que se realizó la nefrectomía radical abierta.

\section{Factores de riesgo:}

1. Obesidad: En nuestros pacientes el IMC de $28,3(21,9-40)$ reflejaba un sobrepeso, con $38 \%$ de Obesidades (IMC 30 o superior). Esto no nos ha ocasionado ningún problema, teniendo unos resultados similares. Hedican et al. ${ }^{31}$ publican la laparoscopia en 30 pacientes con IMC superior a 30 , sin complicaciones mayores, 23,3\% de complicaciones menores, sobre todo íleo paralítico, EM de 4,1 días y tiempo quirúrgico de $262 \mathrm{~min}$. Nosotros no hemos observado que los pacientes con obesidad (13 casos) hayan tenido una EM mayor (5,9 días), ni más complicaciones, ni más tiempo quirúrgico (147 minutos las nefrectomías radicales y 167 minutos las nefroureterectomías), aunque si han tardado más en iniciar dieta oral y en deambular que los no obesos. Tradicionalmente la obesidad se había considerado una con- traindicación relativa; los últimos datos muestran que el índice de complicaciones y los resultados pueden ser similares, precisando sólo un cambio en la colocación de los trócares y una mayor presión de insuflación ${ }^{32}$; por ello no se debe considerar una contraindicación ${ }^{32}$.

2. ASA: Hemos determinado el ASA de los pacientes, teniendo una media de 2,3 (1-4). En pacientes de mayor riesgo, Baldwin et al. ${ }^{33}$ revisan 21 con ASA igual o mayor de 3 operados de nefrectomía laparoscópica (13 laparoscopia convencional y 8 Mano-asistida), y los ASA 4 tenían más EM y gasto hospitalario que los ASA 3, sin diferencias en el resto de los parámetros, y al comparar entre laparoscopia y nefrectomía abierta, el grupo de laparoscopia de alto riesgo tenía menor consumo analgésico, menor EM, menor gasto quirúrgico y mejor incorporación de la dieta oral que el grupo de cirugía abierta, concluyendo que la nefrectomía laparoscópica convencional y la Mano-asistida se pueden realizar en estos pacientes de alto riesgo, con porcentaje de complicaciones del 0-25\%, no significativo por el bajo número ${ }^{33}$.

Nosotros hemos tenido 10 ASA 3 y 1 ASA 4, con IMC algo superior, 30,3 (27,2 en los ASA 12), edad de 68,9 (64,5 en ASA 1-2); con sólo una complicación técnica (la endoGIA atascada), no imputable al ASA, y con el mismo porcentaje de complicaciones menores, $18,2 \%$ por íleo paralítico e insuficiencia cardíaca, sin diferencia en la EM, 6 días (frente a 5,8). Incluso el sangrado medido por la diferencia en la $\mathrm{Hb}$ es similar $(2,6$ por 2,8 del total).

\section{Cirugía abdominal previa}

El 28,6\% habían sido operados de cirugía abdominal. Inicialmente creíamos que tendríamos más dificultades técnicas, pero salvo más adherencias para liberar (aumentan poco el tiempo quirúrgico), y algún caso de dificultad para colocar el anillo del puerto de la mano, no hemos encontrado ningún otro problema. En otras series hay hasta $46 \%$ de antecedentes quirúrgi$\cos ^{17}$.

\section{Enfermedades concomitantes}

El 65,7\% de nuestros pacientes presentaban enfermedades concomitantes. 
Esperábamos problemas en los 2 pacientes con pancreatitis, pero no detectamos ningún problema local.

Las hernias de hiato no provocaron ningún problema anestésico.

El paciente con radioterapia pelviana impidió realizar la desinserción transuretral del uréter intravesical (no se realizó la ureterectomía intravesical por ser mayor de 80 años, tener tumor en pelvis renal, y temer que los tejidos pelvianos más adheridos provocaran morbilidad).

El paciente con aneurisma de aorta que llevaba un "stent" en la arteria renal del riñón afectado nos planteó la dificultad técnica del aneurisma, que no se produjo, y pudimos evitar el "stent"en el momento de poner los clips en la arteria renal, aunque teniamos previsto haber cortado la arteria con el "stent" con una tijera de Metzenbaum a través del Gelport.

\section{Datos histopatológicos}

El tamaño de la pieza quirúrgica de 490 g (195896) en las nefrectomias radicales, 460 g (147853) en las nefroureterectomías y 312 g (50-626) en las nefrectomías simples nos hubiera obligado a abrir al final de la intervención una incisión similar a la del dispositivo de mano-asistida.

El tamaño de los carcinomas renales, $4,8 \mathrm{~cm}$ de media $(2,3-8,5 \mathrm{~cm})$, es similar al de otras series, pero según aumenta la experiencia van aumentando las indicaciones de laparoscopia en base al tamaño. En la literatura hay series con tamaños tumorales medios en $\mathrm{cm}$ de $4,3^{34}, \quad 5,1^{25}, \quad 5,5^{35}, \quad 5,7^{22}$, $6,3^{30}$, o 4,7 en $\mathrm{T} 1$ y 9,2 en $\mathrm{T} 2^{36}$.

Sólo desestimamos por el tamaño un caso de $12,5 \mathrm{~cm}$, con una pieza de $1.865 \mathrm{~g} \mathrm{y}$ otro de $11 \mathrm{~cm}$ con sospecha de invasión local diafragmática. Por el tamaño, se recomienda empezar con los $\mathrm{T} 1$ más pequeños para luego pasar a los T2; según aumenta la experiencia, con los T2 no hay más complicaciones ${ }^{36}$.

Tabla 2

\section{Datos quirúrgicos}

El tiempo quirúrgico ha sido 140 minutos: 130 (80-210) en las nefrectomías radicales, 135 (120-150) en las nefrectomías simples y 163 (80-240) en las nefroureterectomías. En la literatura el tiempo varía mucho, entre 140294 minutos, como se observa en la Tabla 2. Es más rápida la nefroureterectomía con laparoscopia Mano-asistida que la laparoscopia convencional $^{37}$.

La EM ha sido 5,8 días (3-15), estando el 40\% 3-4 días. Los motivos de las estancias más prolongadas han sido: Una lesión arterial mayor con reimplante, íleo paralítico con drenaje linfático prolongado, y fallo en la EndoGIA que precisó reconversión. Hay EM mayor en las nefroureterectomías (tienen más irritación peritoneal), y tardan algo más en la ingesta oral (1 día en nefrectomias simples, 1,7 en nefrectomias radicales y 2 en nefroureterectomías), además de precisar sonda vesical en el postoperatorio que ha retrasado el alta.

En las nefroureterectomías retroperitoneales Mano-asistidas de Kawauchi et al. tenían EM de 13 días (ninguno menor de 8 días), sin referir el motivo de estancias tan prolongadas ${ }^{11}$. En las revisiones hay resultados muy dispares, (la definición de EM es variable), algunos ponen la EM

EM y Tiempo Quirúrgico en las Nefrectomías Mano-asistidas

\begin{tabular}{|c|c|c|c|c|c|}
\hline & Tipo op & № & Edad & EM & $\begin{array}{c}\text { Tiempo Op } \\
\text { (min) }\end{array}$ \\
\hline Nosotros & & 35 & 66 & 5,8 & 140 \\
\hline Patel y Leveille ${ }^{36}$ & Nef Radical (T1) & 50 & 62 & 3,3 & 190 \\
\hline Gaston et al. ${ }^{22}$ & & 30 & 62 & 2 & 140 \\
\hline Nelson y Wolf ${ }^{30}$ & Nef Radical & 22 & 57 & 2,7 & 205 \\
\hline Landman et al. ${ }^{37}$ & Nefroureterectomía & 16 & 66 & 2,5 & 294 \\
\hline López et al. ${ }^{35}$ & Nef Radical & 11 & 61 & 3,1 & 156 \\
\hline Kawauchi et al. ${ }^{11}$ & Nefroureterectomía & 34 & 67 & 13 & 233 \\
\hline Pietrow et al. ${ }^{38}$ & & 36 & 55 & 4,3 & 175 \\
\hline Harano et al. ${ }^{34}$ & Nef Radical & 96 & 61 & 11,4 & 246 \\
\hline Nakada et al. ${ }^{40}$ & Nef Radical & 18 & 63 & 3,9 & 220 \\
\hline Stifelman et al. ${ }^{23}$ & Nef Radical & 74 & & 3,7 & 198 \\
\hline Stifelman et al. ${ }^{23}$ & Nefroureterectomía & 22 & & 4,1 & 272 \\
\hline Wolf et al. ${ }^{17}$ & & 13 & 55 & 3,1 & 240 \\
\hline
\end{tabular}


global, otros la EM de aquellos sin complicaciones, excluyendo las reconversiones, por ello es difícil la comparación.

En nuestros casos la EM en los no complicados ha sido 4,2 días, y en los complicados 9,7.

\section{Complicaciones}

En ningún caso se trasfundió, aunque hubo descensos de la hemoglobina de 2,8 g/1 (13,9 a $11,1)$. Tan et al. muestran $45 \%$ de complicaciones, $15 \%$ mayores, en sus nefrectomías manoasistidas en procesos inflamatorios ${ }^{28}$.

Complicaciones mayores: Tenemos 5,7\%(2) reconversiones, una por fallo en la EndoGIA, en uno de los primeros casos, descrito también en otras publicaciones ${ }^{25}$ y otro por ligadura de la arteria mesentérica superior; y 5,7\% (2) reoperaciones. En estos casos la EM ha aumentado a 11,7 días. La tasa de reconversiones en la literatura es del $0 \% 38,8,7 \%$ en $\mathrm{T}^{36}, 9,4 \%^{2}$, siendo más frecuentes, $12 \%$, en los primeros $25 \operatorname{casos}^{36}$. Tanto Gill et al. ${ }^{39}$ como Rassweiler et al. ${ }^{2}$ encuentran que la mayoría de las complicaciones suceden en las primeras 20 laparoscopias de cada cirujano.

Complicaciones menores: Tenemos 17,1\% (6 pacientes), que han aumentado la EM a 8,8 días.

\section{CONCLUSIONES}

La nefrectomía laparoscópica Mano-asistida es una técnica accesible para la mayoría de urólogos, permitiendo iniciar un programa de laparoscopia con menor curva de aprendizaje y unos resultados eficaces, compartiéndolos con la nefrectomía por laparoscopia convencional.

\section{REFERENCIAS}

1. Clayman RV, Kavoussi LR, Soper NJ, Dierks SM, Meretyk S, Darcy MD, et al. Laparoscopic nephrectomy: initial case report. J Urol. 1991;146(2):278-282.

2. Rassweiler J, Fornara P, Weber M, Janetschek G, Fahlenkamp D, Henkel T, et al. Laparoscopic nephrectomy: the experience of the laparoscopy working group of the German Urologic Association. J Urol. 1998;160(1):18-21.

3. Stifelman M, Nieder AM. Prospective comparison of handassisted laparoscopic devices. Urology. 2002;59(5):668-672.

4. Patel R, Stifelman MD. Hand-assisted laparoscopic devices: The second generation. J Endourol. 2004;18(7):649-653.

5. Boland JP, Kusminsky RE, Tiley EH: Laparoscopic minilaparotomy with manipulation: the middle path. Minim Invasive Ther. 1993;2(6):63-67.

6. Bannenburg JJG, Maijer DW, Bannenburg JH, Hodde KC. Hand-assisted laparoscopic nephrectomy in the pig:inicial report. Minim Invas Ther Allied Tech 1996;5:483.
7. Nakada SY, Moon TD, Gist M, Mahvi D. Use of the PneumoSleeve as an adjunct in laparoscopic nephrectomy. Urology 1997;49(4):612-613.

8. Keeley FX, Sharma NK, Tolley DA: Hand-assisted laparoscopic nephroureterectomy (abstract 1565). J Urol. 1997, 157(suppl):399A.

9. Wolf JS Jr, Moon TD, Nakada SY. Hand-assisted laparoscopic nephrectomy: Technical considerations. Techn Urol.1997;Fall 3(3)3:123-128.

10. Stifelman MD, Sosa RE, Andrade A, Tarantino A, Shichman SJ. Hand-assisted laparoscopic nephroureterectomy for the treatment of transitional cell carcinoma of the upper urinary tract. Urology. 2000;56(5):741-747.

11. Kawauchi A, Fujito A, Ukimura O, Yoneda K, Mizutani Y, Miki T. Hand assisted retroperitoneoscopic nephroureterectomy: comparison with the open procedure. J Urol. 2003;169(3):890-894.

12. Slakey DP, Wood JC, Hender D, Thomas R, Cheng S. Laparoscopic living donor nephrectomy: Advantages of the hand-assited method. Transplantation. 1999;68(4):581583.

13. Stifelman MD, Sosa RE, Nakada SY, Shichman SJ. Hand assisted laparoscopic partial nephrectomy. J Endourol. 2001;15(2):161-164.

14. Strup SE, Hubosky S. Hand assisted laparoscopic partial nephrectomy. J Endourol 2004;18(4):345-349.

15. Strup S, Garrett J, Gomella L, Rowland R. Laparoscopic partial nephrectomy: hand-assisted technique. J Endourol. 2005; 19(4):456-459.

16. Peterson AC, Lance RS, Ahuja S. Laparoscopic hand assisted radical cystectomy with ileal conduit urinary diversion. J Urol. 2002;168(5):2103-2105.

17. Wolf JS, Moon TD, Nakada SY. Hand assisted laparoscopic nephrectomy: comparison to standard laparoscopic nephrectomy. J Urol. 1998;160(1):22-27.

18. Nakada SY. Techniques in Endourology. Hand-assisted laparoscopic nephrectomy. J Endourol. 1999;13(1):9-15.

19. Fadden PT, Stephen Y, Nakada MD. Hand-assisted laparoscopic renal surgery. Urol Clin N Am. 2001;28(1):167176.

20. Munver R, Del Pizzo JJ, Sosa E. The advantages of handassited laparoscopy. Curr Urol Rep. 2004;5(2):100-107.

21. Sosa ER. Hand assisted laparoscopic nephrectomy. Editorial comments. J Endourol. 1999;13(1):14-15.

22. Gaston KE, Moore DT, Pruthi RS. Hand-assisted laparoscopic nephrectomy: prospective evaluation of the learning curve. J Urol. 2004;171(1):63-67.

23. Stifelman MD, Sosa RE, Shichman SJ. Hand-assisted laparoscopy in urology. Rev Urol. 2001;3(2):63-71.

24. Gill IS. Hand assisted laparoscopy:con. Urology. 2001;58: 313-317.

25. Wille AH, Roigas J, Deber S, Tüllmann M, Türk I, Loening SA. Laparoscopic radical nephrectomy: Techniques, results and oncological outcome in 125 consecutive cases. Eur Urol. 2004;45(4):483-488.

26. Kawauchi A, Fujito A, Soh J, Ukimura O, Mizutani Y, Miki T. Learning curve of hand-assisted retroperitoneoscopic nephrectomy in less-experienced laparoscopic surgeons. Int J Urol. 2005; 12(1):1-6.

27. Batler RA, Schoor RA, González CM, Engel JD, Nadler RB. Hand-assisted laparoscopic radical nephrectomy: the experience of the enexperienced. J Endourol. 2001;15(5): 513-516. 
28. Tan YH, Siddiqui K, Preminger GM, Albala DM. Hand assisted laparoscopic nephrectomy for inflammatory renal conditions. J Endourol. 2004;18(8):770-774.

29. Cobb WS, Heniford BT, Matthews BD, Carbonell AM, Kercher KW. Advanced age is not a prohibitive factor in laparoscopic nephrectomy for renal pathology. Am Surg. 2004;70(6):537-542.

30. Nelson CP, Wolf JS Jr. Comparison of hand assisted versus standard laparoscopic radical nephrectomy for suspected renal cell carcinoma. J Urol. 2002;167(5):1989-1994.

31. Hedican SP, Moon TD, Lowry PS, Nakada SY. Hand assisted laparoscopic renal surgery in the morbidly and profoundly obese. J Endourol. 2004;18(3):241-244.

32. Fugita OE, Chan DY, Roberts WW, Kavoussi LR, Jarrete TW. Laparoscopic radical nephrectomy in obese patients: outcomes and technical considerations. Urology. 2004; 63(2):247-252.

33. Baldwin DD, Dunbar JA, Parekh DJ, Wells N, Shuford MD, Cookson MS, et al. Single-center comparison of purely laparoscopic, and open radical nephrectomy in patients at high anesthetic risk. J Endourol. 2003;17(3):161-167.

34. Harano M, Eto M, Omoto K, Tatsugami K, Nomura H, Koga $\mathrm{H}$, et al. Long-term outcome of hand-assisted laparoscopic radical nephrectomy for localizad stage $\mathrm{T} 1 / \mathrm{T} 2$ renal cell carcinoma. J Endourol. 2005;19(7):803-807.

35. Lopez Cubillana P, Prieto González A, Gómez Gómez G, Cao Avellaneda E, López López AI, Maluff Torres A, et al. Nefrectomía Radical Laparoscópica asistida por la mano frente a Nefrectomía abierta en el tratamiento del carcinoma de células renales clínicamente localizado. Estudio comparativo. Arch Esp Urol. 2004;57(8):833-837.
36. Patel VR, Leveillee RJ. Hand-assisted laparoscopic nephrectomy for satge $\mathrm{T} 1$ and large stage $\mathrm{T} 2$ renal tumors. $\mathrm{J}$ Endourol. 2003;17(6):379-383.

37. Landman J, Lev RY, Bhayani S, Alberts G, Rehman J, Pattaras JG, et al. Comparison of hand assisted and standard laparoscopic radical nephroureterectomy for the management of localized transitional cell carcinoma. J Urol. 2002;167(6):2387-2391.

38. Pietrow PK, Auge BK, Terranova S, Ekeruo W, Preminger GM, Albala DM. Hand assisted laparoscopic nephrectomy: the transfer of experience to a new academic center. $J$ Endourol. 2004;18(9):840-843.

39. Gill IS, Kavoussi LR, Clayman RV, Ehrlich R, Evans R, Fuchs G, et al. Complications of laparoscopic nephrectomy in 185 patients: a multi-institutional review. J Urol. 1995; 154(2):479-483.

40. Nakada SY, Fadden P, Jarrard DF, Moon TD. Hand-assisted laparoscopic radical nephrectomy: comparison to open radical nephrectomy. Urol. 2001,58(4):517-520.

Dr. C. Gutiérrez Sanz-Gadea

E-mail: cgut@hsll.es

(Trabajo recibido el 9 de mayo 2006) 